\title{
Results of a sensing system for an autonomous mobile robot based on the paraconsistent artificial neural network
}

\section{Cláudio Rodrigo Torres*}

Faculdade de Exatas e Tecnologia - FACET,

Universidade Metodista de São Paulo,

São Bernardo do Campo, SP, Brazil

Email: c.r.t@uol.com.br

*Corresponding author

\section{Germano Lambert-Torres}

Artificial Intelligence Application Group - GAIA, Federal University of Itajubá, Av. BPS 1303 - 37.500-903, Itajubá, MG, Brazil

Email: germanoltorres@gmail.com

\section{João Inácio Da Silva Filho}

Department of Electric Engineering,

Santa Cecilia University - UNISANTA, Oswaldo Cruz street, 266 - Santos City, SP, Brazil

Email: inacio@unisanta.br

\section{Helga Gonzaga Martins}

Artificial Intelligence Application Group - GAIA, Federal University of Itajubá, Av. BPS 1303 - 37.500-903, Itajubá, MG, Brazil Email: helgagonzaga@gmail.com

\begin{abstract}
This paper shows the results of a sensing system for an autonomous mobile robot. The sensing system is based on the paraconsistent neural network. The type of artificial neural network used in this work is based on the paraconsistent evidential logic $(\mathrm{E} \tau)$. The objective of the sensing system is to inform the other robot components the obstacle position. The reached results have been satisfactory.
\end{abstract}

Keywords: paraconsistent neural network; paraconsistent logic; autonomous mobile robot; sensing system.

Reference to this paper should be made as follows: Torres, C.R., Lambert-Torres, G., Da Silva Filho, J.I. and Martins, H.G. (2011) 'Results of a sensing system for an autonomous mobile robot based on the paraconsistent artificial neural network', Int. J. Reasoning-based Intelligent Systems, Vol. 3, No. 2, pp.108-114.

Biographical notes: Cláudio Rodrigo Torres received the $\mathrm{PhD}$ and $\mathrm{MSc}$ degrees from Itajuba Federal University (2010), Brazil, and the BSc (2000 and 2004)) degree from Santa Cecilia University, Brazil, all in Electric Engineering. He works as Professor at Universidade Metodista de São Paulo, Brazil.

Germano Lambert-Torres received the BSc and MSc degrees from Itajuba Federal University, Brazil, and the PhD degree from Ecole Polytechnique at Montreal, all in Electrical Engineering in 1982, 1986 and 1990, respectively. He also received a BSc degree in Mathematics from the Itajuba Faculty of Philosophy, Sciences and Letters, both in 1981. Currently, he works as full Professor at Itajuba Federal University. He has more than 500 journals and conference published papers and more than 70 master and $\mathrm{PhD}$ supervisions to his credit. His research interests are in the areas of power system operation and intelligent system applications.

João Inácio Da Silva Filho received the $\mathrm{PhD}$ in Electric Engineering from the Polytechnic School of São Paulo University (POLI/USP) in the area of Digital Systems and the master's degree in Microelectronic for the same Institution. He is the Coordinator of the Paraconsistent Applied 
Logic Group (GLPA) and member of the Group of Logic and Theory of the Science of (IEA) Institute of Advanced Studies of São Paulo University. He was the first autonomous robot's creator capable to work with a paraconsistent logical controller (Robot Emmy), and now is developing researches about the paraconsistent artificial networks applications in expert systems.

Helga Gonzaga Martins received the BSc and MSc degrees in Mechanical Engineering and the $\mathrm{PhD}$ degree in Electrical Engineering from Itajuba Federal University in 1994, 1998 and 2003, respectively. Her research interests are in the areas of classical logic, paraconsistent logic, and artificial intelligence applications.

\section{Introduction}

This paper describes the architecture of an autonomous mobile robot which is able to achieve a set point in an environment divided into coordinates (Torres, 2010). In this work, an autonomous mobile robot is considered as a system divided into three other subsystems: planning subsystem, sensing subsystem and mechanical subsystem. The planning subsystem is responsible for generating the sequence of movements the robot must perform to achieve a set point. The sensing subsystem has the objective of informing the planning subsystem the position of obstacles; and the mechanical subsystem is the robot itself, it means the mobile mechanical platform which carries all devices from the other subsystems. This platform must also perform the sequence of movements borne by the planning subsystem.

The planning subsystem and the sensing subsystem have already been implemented, but the mechanical subsystem has not been implemented yet. The sensing subsystem uses the paraconsistent artificial neural network (Da Silva Filho et al., 2008, 2010). This type of artificial neural network is based on the paraconsistent evidential logic $(E \tau)$. We describe some concepts about $E \tau$ in the next section.

This paper focuses on the sensing system of the robot (Torres et al., 2010).

\section{Paraconsistent evidential logic}

The $\mathrm{E} \tau$ (Abe, 1992) is a type of paraconsistent logic in which there may be a favourable evidence degree $(\mu)$ and a contrary evidence degree $(\lambda)$ in each analysed sentence.

The $\mu$ is a value between 0 and 1 that represents the favourable evidence in which the sentence is true.

The $\lambda$ is a value between 0 and 1 that represents the contrary evidence in which the sentence is true.

Through the favourable and contrary degrees, it is possible to represent the four extreme logic states as shown in Figure 1.

The four extreme logic states are:

- $\quad$ true $(\mathrm{V})$

- false (F)

- paracomplete $(\perp)$

- inconsistent (T).
Da Silva Filho (1999) proposed the paranalyser algorithm. By this algorithm, it is also possible to represent the nonextreme logic state. Figure 2 shows this.

Figure 1 Extreme logic states

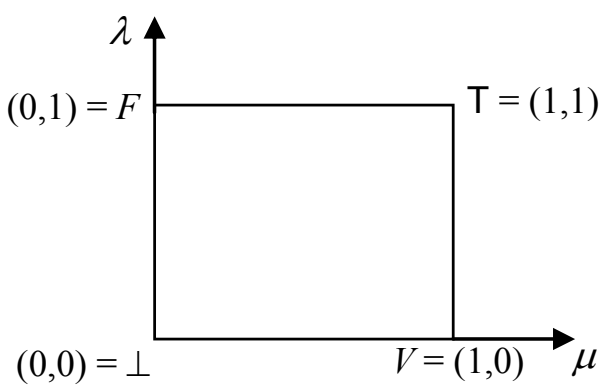

The eight non-extreme logic states are:

- quasi-true tending to inconsistent $-\mathrm{QV} \rightarrow \mathrm{T}$

- quasi-true tending to paracomplete $-\mathrm{QV} \rightarrow \perp$

- $\quad$ quasi-false tending to inconsistent $-\mathrm{QF} \rightarrow \mathrm{T}$

- quasi-false tending to paracomplete $-\mathrm{QF} \rightarrow \perp$

- quasi-inconsistent tending to true $-\mathrm{QT} \rightarrow \mathrm{V}$

- quasi-inconsistent tending to false $-\mathrm{QT} \rightarrow \mathrm{F}$

- quasi-paracomplete tending to true $-\mathrm{Q} \perp \rightarrow \mathrm{V}$

- quasi-paracomplete tending to false $-\mathrm{Q} \perp \rightarrow \mathrm{F}$.

Figure 2 Non-extreme logic states

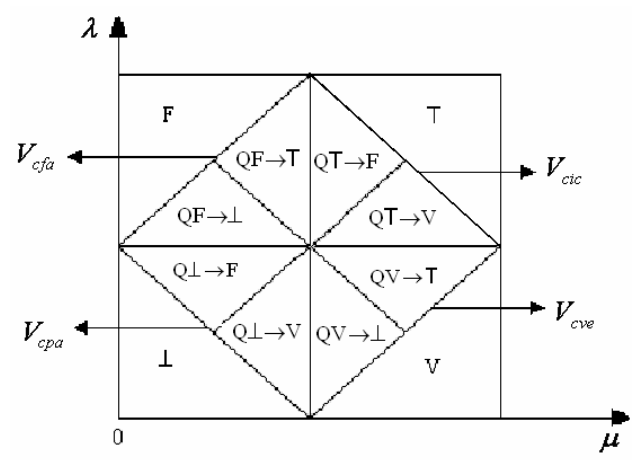

The uncertainty degree is given as $G_{\mathrm{un}}(\mu, \lambda)=\mu+\lambda-1$ and the certainty degree is given as $G_{\mathrm{c}}(\mu, \lambda)=\mu-\lambda(0 \leq \mu$, $\lambda \leq 1)$ 
Some additional control values are:

- $\quad V_{\text {cic }}=$ maximum value of uncertainty control

- $\quad V_{\mathrm{cve}}=$ maximum value of certainty control

- $\quad V_{\mathrm{cpa}}=$ minimum value of uncertainty control

- $\quad V_{\text {cfa }}=$ minimum value of certainty control.

In the next section, we describe the proposed sensing system.

\section{Sensing system}

The sensing system is a set of electronic components and software responsible for analysing the environment around the robot and detecting the obstacle positions. After that, it must inform the other components of the robot the position of the obstacles.

The sensing system may get information from any type of sensor. It is shown in this paper that a sensing system is able to deal with information from just one ultrasonic sensor. But, if there is more than one sensor in the robot, it is possible to build a sensing system similar to the one shown here for each sensor.

Elfes (1989) presented a method of robot perception and world's modelling that uses a probabilistic tessellated representation of spatial information called the Occupancy Grid. A similar method is proposed here, but instead of using probabilistic representation, the paraconsistent evidential logic $(\mathrm{E} \tau)$ is used.

The proposed sensing system aims to generate a favourable evidence degree in each environment position. The favourable evidence degree is related to the sentence: there is obstacle in the analysed position.

The sensing system is divided into two parts. The first part is responsible for receiving the data from the sensors and sending information to the second part of the system. And the second part is the paraconsistent artificial neural network itself. Figure 3 shows this idea.

Figure 3 Representation of the sensing system

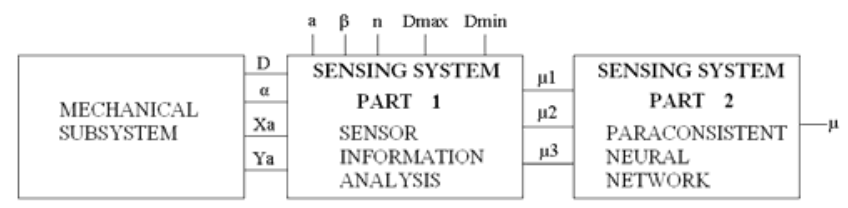

The proposed sensing system is prepared to receive data from ultrasonic sensors. The robot sensors are on the mechanical subsystem. So, this subsystem must treat the data generated by the sensors and send information to the first part of the sensing subsystem. The data the mechanical subsystem must send to the first part of the sensing subsystem are: $D, \alpha, X_{\mathrm{a}}$ and $Y_{\mathrm{a}}$.

1 The distance between the sensor and the obstacle $(D)$.

2 The angle between the horizontal axis of the environment and the direction to the front of the sensor $(\alpha)$. Figure 4 shows the angle $\alpha$.

3 The coordinate where the robot is $\left(X_{\mathrm{a}}, Y_{\mathrm{a}}\right)$.
In the first part of the sensing subsystem, there are also some configuration parameters. They are:

1 Figure 5 shows the distance between the environment coordinates $(a)$.

2 Figure 6 shows the angle of the ultrasonic sensor conical field of view $(\beta)$.

3 The number of positions on the arch BC, shown in Figure 6, considered by the system $(n)$.

4 The maximum distance measured by the sensor which the system considers $\left(D_{\max }\right)$.

5 The minimum distance measured by the sensor which the system considers $\left(D_{\min }\right)$.

Figure 4 Angle $\alpha$

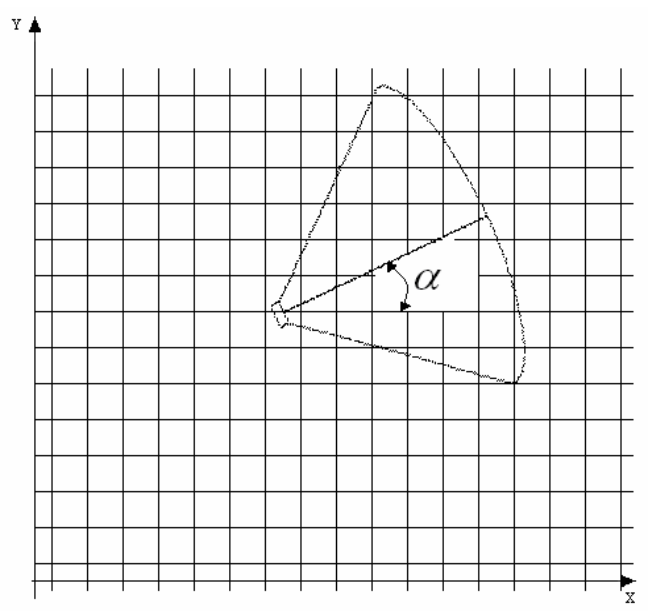

Figure 5 Distance between coordinates

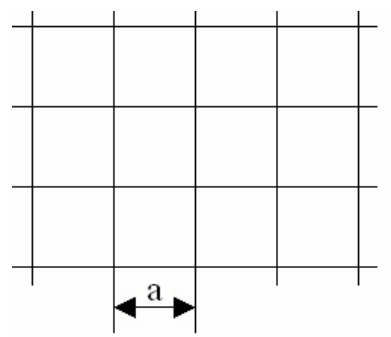

Figure 6 Ultrasonic sensor conical field of view $(\beta)$

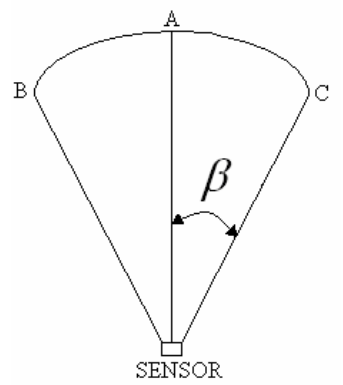

The first part of the sensing system generates three favourable evidence degrees, $\mu_{1}, \mu_{2}$ and $\mu_{3}$.

The favourable evidence degree $\mu_{1}$ is related to the distance between the sensor and the obstacle. The nearer the obstacle is to the sensor, the bigger the $\mu_{1}$ value is. 
The favourable evidence degree $\mu_{2}$ is related to the coordinate position on the arch BC shown in Figure 6 . The nearer the analysed coordinate is to the point $\mathrm{A}$, the bigger is the $\mu_{2}$ value. And the nearer the analysed coordinate is to the points $\mathrm{B}$ and $\mathrm{C}$, the smaller is the $\mu_{2}$ value. The inspiration of this idea comes from Boreinstein's (1991) study which says that the probability of the obstacle near to the point $\mathrm{A}$ is high. And this probability decreases as we analyse the region near to the points $\mathrm{B}$ and $\mathrm{C}$.

Eventually, the favourable evidence degree $\mu_{3}$ is the previous value of the coordinate of favourable evidence degree.

\section{Paraconsistent artificial neural network}

The sensing subsystem neural network of the robot is composed of two types of cells: Analytic Paraconsistent Artificial Neural Cell (CNAPa) and Passage Paraconsistent Artificial Neural Cell (CNAPpa). Below, the cells are described.

\subsection{Analytic paraconsistent artificial neural cell}

This cell has two inputs $\left(\mu_{\mathrm{RA}}\right.$ and $\left.\mu_{\mathrm{RB}}\right)$ and two outputs (S1 and $\mathrm{S} 2)$. Also there are two configuration parameter inputs $\left(F t_{\mathrm{ct}}\right.$ and $F t_{\mathrm{c}}$ ). Figure 7 shows the graphic representation of this cell.

Figure 7 Graphic representation of the analytic paraconsistent artificial neural cell

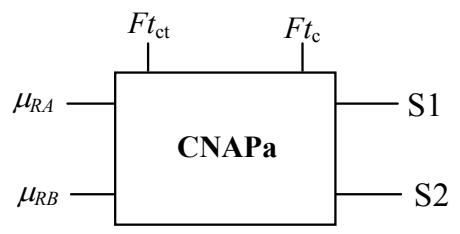

The input evidence degrees are:

$$
\begin{aligned}
& \mu_{\mathrm{RA}}, \text { such as } 0 \leq \mu_{\mathrm{RA}} \leq 1 \\
& \mu_{\mathrm{RB}}, \text { such as } 0 \leq \mu_{\mathrm{RB}} \leq 1
\end{aligned}
$$

There are also two control values:

Contradiction tolerance factor $\left(F t_{\mathrm{ct}}\right)$, such as $0 \leq F t_{\mathrm{ct}} \leq 1$

Certainty tolerance factor $\left(F t_{\mathrm{c}}\right)$, such as $0 \leq F t_{\mathrm{c}} \leq 1$

The CNAPa has two outputs. The output 1 (S1) is the resultant evidence degree $\left(\mu_{\mathrm{E}}\right)$.

$$
\mu_{\mathrm{E}} \text {, such as } 0 \leq \mu_{\mathrm{E}} \leq 1
$$

The output $2(\mathrm{~S} 2)$ is the resultant evidence interval $\left(\varphi_{\mathrm{E}}\right)$.

$$
\varphi_{\mathrm{E}} \text {, such as } 0 \leq \varphi_{\mathrm{E}} \leq 1
$$

The CNAPa calculates the maximum value of certainty $\left(V_{\text {cve }}\right)$, the minimum value of certainty control $\left(V_{\text {cfa }}\right)$, the maximum value of uncertainty control $\left(V_{\text {cic }}\right)$ and the minimum value of uncertainty control $\left(V_{\text {cpa }}\right)$ by the following equations:

$$
\begin{aligned}
& V_{\mathrm{cve}}=\frac{1+F t_{\mathrm{c}}}{2} \\
& V_{\mathrm{cfa}}=\frac{1-F t_{\mathrm{c}}}{2}
\end{aligned}
$$

$$
\begin{aligned}
& V_{\text {cic }}=\frac{1+F t_{\mathrm{ct}}}{2} \\
& V_{\mathrm{cpa}}=\frac{1-F t_{\mathrm{ct}}}{2}
\end{aligned}
$$

The $\mu_{\mathrm{E}}$ is determined in the following equation:

$$
\mu_{\mathrm{E}}=\frac{G_{\mathrm{c}}+1}{2}
$$

As $G_{\mathrm{c}}=\mu-\lambda$, we can say that:

$$
\mu_{\mathrm{E}}=\frac{\mu-\lambda+1}{2}
$$

It is called as certainty interval $(\varphi)$, the certainty degree interval that can be modified without changing the uncertainty degree value. This value is determined in the below equation:

$$
\varphi=1-\left|G_{\mathrm{ct}}\right|
$$

\subsection{Passage paraconsistent artificial neural cell}

The CNAPpa has one input $(\mu)$, one output (S1) and one parameter control input $\left(F t_{\mathrm{c}}\right)$. Figure 8 shows the graphic representation of CNAPpa.

Figure 8 Graphic representation of the passage paraconsistent artificial neural cell

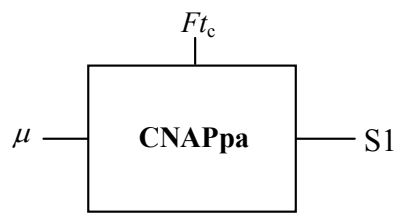

The input is $\mu$.

$$
\mu \text {, such as } 0 \leq \mu \leq 1
$$

The value of the output $\mathrm{S} 1$ is the same as the input $\mu$. But the output value may be limited through the parameter control input $F t_{\mathrm{c}}$.

The CNAPpa calculates the maximum value of certainty ( $\left.V_{\text {cve }}\right)$ and the minimum value of certainty control $\left(V_{\text {cfa }}\right)$ by the following equations:

$$
\begin{aligned}
& V_{\mathrm{cve}}=\frac{1+F t_{\mathrm{c}}}{2} \\
& V_{\mathrm{cfa}}=\frac{1-F t_{\mathrm{c}}}{2}
\end{aligned}
$$

It also determines the $\mu_{\mathrm{E}}$ by the following equations:

$$
\begin{aligned}
& \mu_{\mathrm{E}}=\frac{\mu-\lambda+1}{2} \\
& \lambda=1-\mu
\end{aligned}
$$

The output S1 assumes the same value as in the input $\mu$ when the following situation is true:

$$
\left[\left(V_{\text {cve }} \leq \mu_{\mathrm{E}}\right) \text { or }\left(\mu_{\mathrm{E}} \leq V_{\text {cfa }}\right)\right]
$$

Otherwise, $\mathrm{S} 1$ is 0.5 . 


\subsection{Paraconsistent artificial neural architecture}

In Figure 9, it is possible to see the chosen paraconsistent neural network architecture for the sensing subsystem.

Figure 9 The chosen paraconsistent neural network architecture for the sensing system

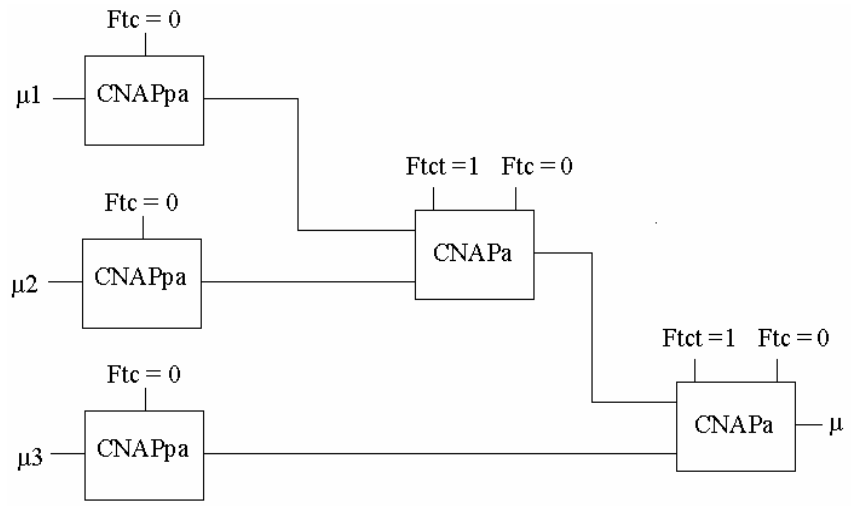

\section{Reached results}

The sensing system has been tested by simulating its inputs and analysing the database generated. The database stores the favourable evidence degree in each environment position analysed. The result of three tests is shown here. The information from one ultrasonic sensor was considered as the sensing system inputs.

\subsection{First test}

The configuration parameters of this test were as follows. The distance between the environment coordinates $(a)$ is 10 . The angle of the ultrasonic sensor conical field of view $(\beta)$ is 30 . The number of positions on the arch of the sensor conical field of view considered by the system $(n)$ is 10 . The maximum distance measured by the sensor which the system considers $\left(D_{\max }\right)$ is 800 . The minimum distance measured by the sensor which the system considers $\left(D_{\min }\right)$ is 8 .

The mechanical subsystem treats the data from the sensors and generates the sensing subsystem inputs. It was needed to simulate the sensing subsystem inputs because the mechanical subsystem has not been implemented yet.

Thus, the simulated sensing subsystem data were the ones described as follows. The distance between the sensor and the obstacle $(D)$ is 200 . The angle between the horizontal axis of the environment and the direction to the front of the sensor $(\alpha)$ is 30 . The coordinate where the $\operatorname{robot}\left(X_{\mathrm{a}}, Y_{\mathrm{a}}\right)$ is $(0,0)$.

We simulated the first measuring of the sensor, when $\mu_{3}$ was initially 0 .

Figure 10 shows the representation of the coordinates in which the sensing system is considered to have obstacles in. Summarising, Figure 10 is a graphical representation of the database generated by the sensing subsystem.
Figure 10 The graphical representation of the database generated by the first test of the sensing subsystem

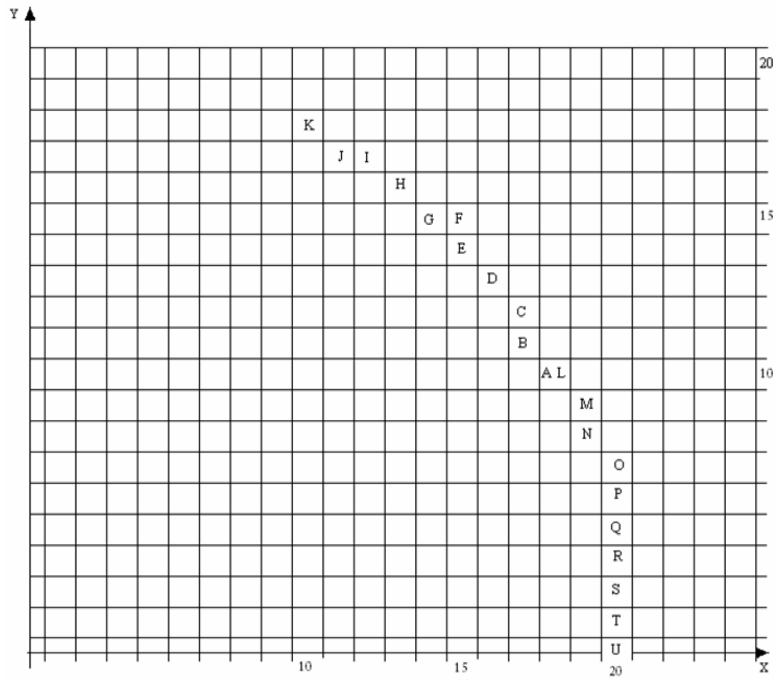

\subsection{Second test}

The configuration parameters of this test were the same as the ones from the first test. The simulated sensing subsystem data were the ones described as follows. The distance between the sensor and the obstacle $(D)$ is 400 . The angle between the horizontal axis of the environment and the direction to the front of the sensor $(\alpha)$ is 45 . The coordinate where the robot $\left(X_{\mathrm{a}}, Y_{\mathrm{a}}\right)$ is $(0,0)$.

We simulated the first measuring of the sensor, when, $\mu_{3}$ was initially 0 . Figure 11 shows the graphical representation of the database generated by the sensing subsystem.

Figure 11 The graphical representation of the database generated by the second test of the sensing subsystem

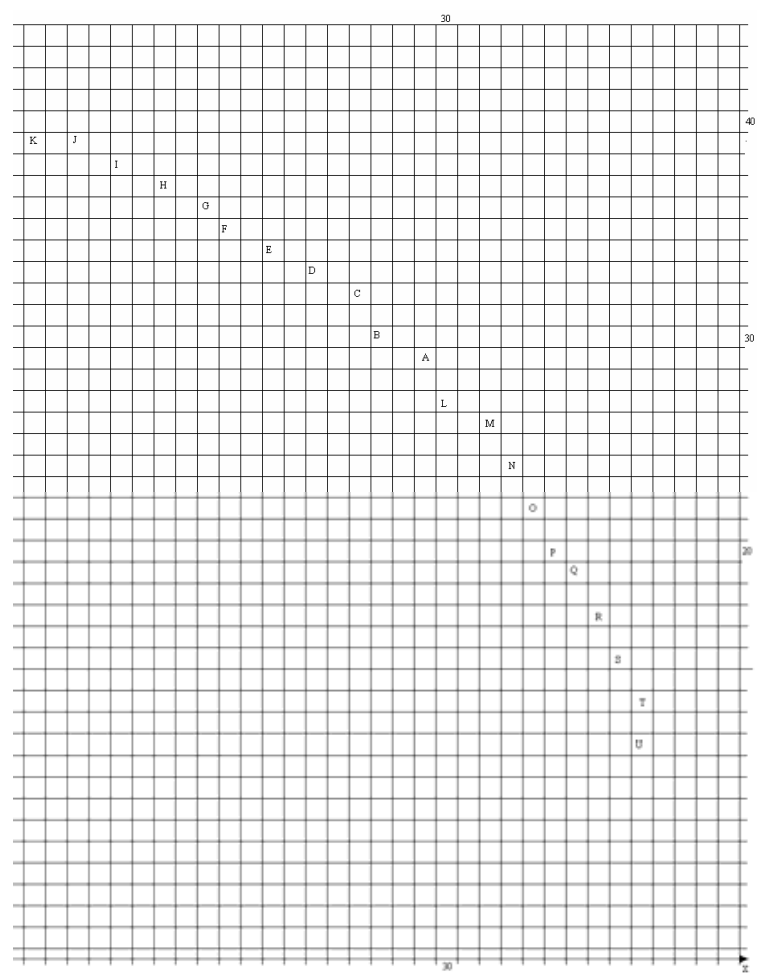


The analysed coordinates and their favourable evidence degree for first test are shown in Table 1.

Table 1 Results of the first test

\begin{tabular}{ll}
\hline Coordinate & $\mu$ \\
\hline A $(18,10)$ & 0.438 \\
B $(17,11)$ & 0.413 \\
C $(17,12)$ & 0.388 \\
D $(16,13)$ & 0.363 \\
E $(15,14)$ & 0.338 \\
F $(15,15)$ & 0.313 \\
G $(14,15)$ & 0.288 \\
H $(13,16)$ & 0.263 \\
I $(12,17)$ & 0.238 \\
J $(11,17)$ & 0.213 \\
K $(10,18)$ & 0.188 \\
L $(18,10)$ & 0.413 \\
M $(19,9)$ & 0.388 \\
N $(19,8)$ & 0.363 \\
O $(20,7)$ & 0.338 \\
P $(20,6)$ & 0.313 \\
Q $(20,5)$ & 0.288 \\
R $(20,4)$ & 0.263 \\
S $(20,3)$ & 0.238 \\
T $(20,2)$ & 0.213 \\
U $(20,0)$ & 0.188 \\
\hline
\end{tabular}

The analysed coordinates and their favourable evidence degree for second test are shown in Table 2.

Table 2 Results of the second test

\begin{tabular}{lc}
\hline Coordinate & $\mu$ \\
\hline A $(29,29)$ & 0.375 \\
B $(27,30)$ & 0.35 \\
C $(26,32)$ & 0.325 \\
D $(24,33)$ & 0.3 \\
E $(22,34)$ & 0.275 \\
F $(20,35)$ & 0.25 \\
G $(19,36)$ & 0.225 \\
H $(17,37)$ & 0.2 \\
I $(15,38)$ & 0.175 \\
J $(13,39)$ & 0.15 \\
K $(11,39)$ & 0.125 \\
L $(30,27)$ & 0.35 \\
M $(32,26)$ & 0.325 \\
N $(33,24)$ & 0.3 \\
O $(34,22)$ & 0.275 \\
P $(35,20)$ & 0.25 \\
Q $(36,19)$ & 0.225 \\
R $(37,17)$ & 0.2 \\
S $(38,15)$ & 0.175 \\
T $(39,13)$ & 0.15 \\
U $(39,11)$ & 0.125 \\
\hline
\end{tabular}

\subsection{Third test}

The configuration parameters and the sensing subsystem data were the same as the ones from the second test; then the analysed coordinates were the same as the second test. The third test has been done just after the second; therefore, their favourable evidence degrees were different from the second test because $\mu_{3}$ was the favourable evidence degree generated by the second test.

The analysed coordinates and their favourable evidence degree are shown in Table 3.

If it is considered the sequence of positions from $\mathrm{K}$ to $\mathrm{U}$ as an arch in the three tests, it is perceived that the $\mu$ decreases as the coordinate is farther distant from the centre of the arch. It means that the system is working as desired.

Table 3 Results of the third test

\begin{tabular}{lc}
\hline Coordinate & $\mu$ \\
\hline A $(29,29)$ & 0.565 \\
B $(27,30)$ & 0.525 \\
C $(26,32)$ & 0.49 \\
D $(24,33)$ & 0.45 \\
E $(22,34)$ & 0.415 \\
F $(20,35)$ & 0.375 \\
G $(19,36)$ & 0.34 \\
H $(17,37)$ & 0.3 \\
I $(15,38)$ & 0.265 \\
J $(13,39)$ & 0.225 \\
K $(11,39)$ & 0.19 \\
L $(30,27)$ & 0.525 \\
M $(32,26)$ & 0.49 \\
N $(33,24)$ & 0.45 \\
O $(34,22)$ & 0.415 \\
P $(35,20)$ & 0.375 \\
Q (36,19) & 0.34 \\
R (37,17) & 0.3 \\
S $(38,15)$ & 0.265 \\
T $(39,13)$ & 0.225 \\
U $(39,11)$ & 0.19 \\
\hline
\end{tabular}

\section{Conclusions}

This paper presents a proposal of an autonomous mobile robot composed of three modules: sensing subsystem, planning subsystem and mechanical subsystem. The mechanical subsystem has not been implemented yet. The sensing subsystem is emphasised here.

The aim of the sensing subsystem is to inform the planning subsystem the positions in which they may have obstacles in. It considers the environment divided into coordinates. The 
sensing subsystem is based on the paraconsistent artificial neural network. The sensing subsystem neural network is composed of two types of cells: CNAPa and CNAPpa.

The output of the sensing subsystem is the favourable evidence degree related to the sentence: there is obstacle in the position. In fact, the sensing subsystem generates a database with the favourable evidence degree for each analysed coordinate.

Some tests were made with the sensing subsystem. The reached results were satisfactory. The next step is the implementation of the mechanical subsystem and the connection of the three subsystems.

\section{References}

Abe, J.M. (1992) Fundamentos da Lógica Anotada (Foundations of Annotated Logics), PhD Thesis, University of São Paulo, São Paulo. [in Portuguese]

Boreinstein, J. and Koren, Y. (1991) 'The Vector field Histogram: fast obstacle avoidance for mobile robots', IEEE Journal of Robotics and Automation, Vol. 7, pp.278-288.
Da Silva Filho, J.I. (1999) Métodos de Aplicações da Lógica Paraconsistente Anotada de Anotação com Dois Valores LPA2v com Construção de Algoritmo e Implementação de Circuitos Eletrônicos, $\mathrm{PhD}$ Thesis, University of São Paulo, São Paulo. [in Portuguese]

Da Silva Filho, J.I., Abe, J.M., and Lambert-Torres, G. (2008) Inteligência Artificial com Redes de Análises Paraconsistentes: Teoria e Aplicação,LTC, Rio de Janeiro.

Da Silva Filho, J.I., Lambert-Torres, G. and Abe, J.M. (2010) Uncertainty Treatment Using Paraconsistent Logic, Vol. 1, 1st ed., IOS Press, Amsterdam.

Elfes, A. (1989) 'Using occupancy grids for mobile robot perception and navigation', Computer, Vol. 22, No. 6, pp.46-57.

Torres, C.R. (2010) Sistema Inteligente Baseado na Lógica

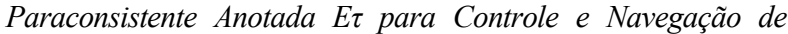
Robôs Móveis Autônomos em um Ambiente Não Estruturado, $\mathrm{PhD}$ Thesis, Federal University of Itajubá, Itajubá, Brazil. [in Portuguese]

Torres, C.R., Abe, J.M., Lambert-Torres, G., Da Silva Filho, J.I. and Martins, H.G. (2010) A Sensing System for an Autonomous Mobile Robot Based on the Paraconsistent Artificial Neural Network, Lecture Notes in Computer Science, Vol. 6278, Springer-Verlag, Berlin/Heidelberg, pp.154-163. 